\title{
On the Automorphism Group of Distinct Weight Codes
}

\author{
Abdelfattah Haily, Driss Harzalla \\ Department of Mathematics, Faculty of Sciences, University Chouaib Doukkali, El Jadida, Morocco \\ Email: afhaily@yahoo.fr, drissHarzalla@yahoo.ca
}

Received 15 January 2015; accepted 24 March 2015; published 25 March 2015

Copyright (C) 2015 by authors and Scientific Research Publishing Inc.

This work is licensed under the Creative Commons Attribution International License (CC BY).

http://creativecommons.org/licenses/by/4.0/

(c) (i) Open Access

\begin{abstract}
In this work, we study binary linear distinct weight codes (DW-code). We give a complete classification of $[n, k]_{2}$-DW-codes and enumerate their equivalence classes in terms of the number of solutions of specific Diophantine Equations. We use the Q-extension program to provide examples.
\end{abstract}

\section{Keywords}

Distinct (Constante) Weigth Code, Automorphism Group, Extension Theorem of MacWilliams, Diophantine Equations

\section{Preliminaries}

One of the main objective of algebraic coding theory is to classify codes up to equivalence by using a list of invariants. The present work is following this way. We study here a class of linear binary codes whose all codewords have distinct weight and will give a classification theorems. Throughout this work all codes are linear binary codes. We call an $[n, k]_{2}$-binary code every $k$ dimensional subspace $\mathcal{C}$ of $\mathbb{F}_{2}^{n}$. Recall also that the Hamming weight $w t(x)$ of vector $x$ is defined to be the number of nonzero components of $x$. The minimum of weights where $x \neq 0$ is the minimal distance $d$ of the code.

A Hamming isometry of $\mathbb{F}_{2}^{n}$ is a linear application $\sigma: \mathbb{F}_{2}^{n} \rightarrow \mathbb{F}_{2}^{n}$ such that $w t(\sigma(x))=w t(x)$, for every $x \in \mathbb{F}_{2}^{n}$. It is well known that in binary case, the isometries are merely the permutations of the coordinates, that is the elements of $\mathcal{S}_{n}$, the permutation group of $\{1,2, \cdots, n\}$.

Two codes $\mathcal{C}$ and $\mathcal{C}^{\prime}$ are said to be equivalent if there exists an isometry $\sigma$ of $\mathbb{F}_{2}^{n}$ such that $\sigma(\mathcal{C})=\mathcal{C}^{\prime}$. An automorphism of $\mathcal{C}$ is a Hamming isometry $\sigma$ such that $\sigma(\mathcal{C})=\mathcal{C}$. The automorphisms of $\mathcal{C}$ form a subgroup of $\mathcal{S}_{n}$ called the automorphism group of $\mathcal{C}$ and we denote it by $\operatorname{Aut}(\mathcal{C})$. Note also that the vector space $\mathbb{F}_{2}^{n}$ can be endowed with a product $\left(x_{1}, \cdots, x_{n}\right) \cdot\left(y_{1}, \cdots, y_{n}\right)=\left(x_{1} y_{1}, \cdots, x_{n} y_{n}\right)$, so that $\left(\mathbb{F}_{2}^{n},+, \cdot\right)$ becomes 
a Boolean ring. Furthermore, $w t(x+y)=w t(x)+w t(y)-2 w t(x y)$, for every $x, y \in \mathbb{F}_{2}^{n}$. The code $\mathcal{C}$ is said a constant-weight code (CW-code) if all nonzero codewords have the same weight. The dual of binary Hamming codes $H_{2}(m)$ are simplex codes $\Sigma_{m}$ of parameters $\left[2^{m}-1, m\right]_{2}$. simplex codes $\Sigma_{m}$ are constant weight code (CW-code).

Any permutation of the columns of a k by n binary matrix $G$ which maps the rows of $G$ into rows of the same matrix, is called an automorphism of the binary matrix $G$ [1]. The set of all automorphisms of $G$ is a subgroup of the symmetric group $S_{n}$ and we denote it by $\operatorname{Aut}(G)$. More treatment of linear codes can be found in the book [2].

Ideally, we would like the rate $R=\frac{k}{n}$ to be high, in order to be able to send a large number of errors. The rate of a DW-code approch zero very quickly when the code length increase: $\frac{k}{n} \leq \frac{1}{n}\left[\frac{\ln (n+1)}{\ln (2)}\right] \searrow 0$ as shown in Figure 1 where $R=\frac{k}{n}$ and $r(k)=\frac{k}{2^{k}-1}$, so $R \leq r(k)$.

It is more convenient to use the DW-codes in the construction of other codes by using some technic of construction and not to use it alone.

\section{Distinct Weight Codes}

Definition 1 A linear binary code $\mathcal{C}$ of length $n$ is said to be a Distinct Weight Code, (in short: $D W$-code), if the weight mapping: wt: $\mathcal{C} \rightarrow\{0,1, \cdots, n\}$, is one to one, that is $x=y$ whenever $w t(x)=w t(y), \forall x, y \in \mathcal{C}$.

The simplest example of such codes are the repetition codes. Later we shall give more nontrivial examples. Let $\mathcal{C}$ a DW-code of length $n$ and dimension $k$. Since the number of element of $\mathcal{C}$ is $2^{k}$, then we have $2^{k} \leq n+1$. In the sequel we fix our interest to the extreme case $2^{k}=n+1$, in which we give a construction.

Proposition 2 Let $k$ such that $2^{k} \leq n+1$. Then every family $u_{1}, \cdots, u_{k} \in \mathbb{F}_{2}^{n}$ of words such that $w t\left(u_{r}\right)=2^{r-1}$ is linearly independent.

Proof. Suppose on the contrary that $u_{1}, \cdots, u_{k}$ are not linearly independent, then we have a linear combination $\sum_{i=1}^{k} \alpha_{i} u_{i}=0$, where some $\alpha_{i}$ is nonzero. Let $r$ be the maximal integer such that $\alpha_{r} \neq 0$. Then $\alpha_{r}=1$, and $u_{r}=\sum_{i=1}^{r-1} \alpha_{i} u_{i}$. Now taking the weights leads to:

$$
2^{r-1}=w t\left(u_{r}\right) \leq \sum_{i=1}^{r-1} w t\left(\alpha_{i} u_{i}\right) \leq \sum_{i=1}^{r-1} 2^{i-1}=2^{r-1}-1
$$

a contradiction.

Now we give a construction of a $\left[2^{k}-1, k\right]$ DW-code.

Let $k$ be a nonzero integer and $n=2^{k}-1$. Take $\left(e_{1}, \cdots, e_{n}\right)$ the canonical basis of $\mathbb{F}_{2}^{n}$. Put $c_{r}=\sum_{i=2^{r-1}}^{2^{r}-1} e_{i}$, then clearly $w t\left(c_{r}\right)=2^{r-1}$. By the proposition 2, the code-words $c_{1}, c_{1}, \cdots, c_{k}$ are linearly independent and generate a $[n, k]$ linear code that we denotes by $\mathcal{D}(k)$. It also seen that $c_{i} c_{j}=0$, whenever $i \neq j$. This

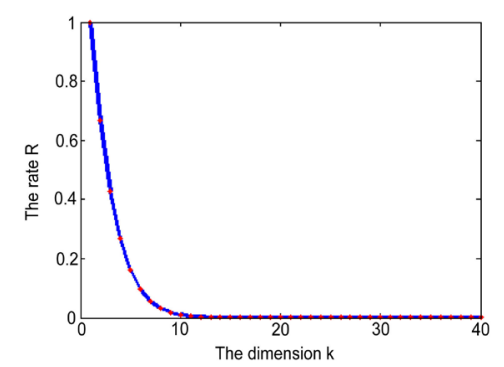

Figure 1. $R \leq r(k)$ where $r(k) \searrow 0$. 
implies that $w t\left(\sum_{i=1}^{k} \alpha_{i} c_{i}\right)=\sum_{i=1}^{k} w t\left(\alpha_{i} c_{i}\right)=\sum_{i=1}^{k} \alpha_{i} 2^{i-1}$.

A generator matrix of $\mathcal{D}(k)$ looks like:

$$
G_{k}=\left(\begin{array}{ccccccccccccc}
1 & 0 & 0 & 0 & 0 & 0 & 0 & 0 & 0 & 0 & \cdots & 0 & 0 \\
0 & 1 & 1 & 0 & 0 & 0 & 0 & 0 & 0 & 0 & \cdots & 0 & 0 \\
0 & 0 & 0 & 1 & 1 & 1 & 1 & 0 & 0 & 0 & \cdots & 0 & 0 \\
\vdots & \vdots & \vdots & \vdots & \vdots & \vdots & \vdots & \vdots & \vdots & \vdots & \vdots & \vdots & \vdots \\
0 & 0 & 0 & 0 & 0 & 0 & 0 & 0 & 0 & 1 & \cdots & 1 & 1
\end{array}\right)
$$

Proposition 3 The $\left[2^{k}-1, k\right]$-code $\mathcal{D}(k)$ is a DW-code.

Proof. Since the cardinal of $\mathcal{D}(k)$ is $2^{k}=n+1$, it suffices to show that wt: $\mathcal{D}(k) \rightarrow\{0,1, \cdots, n\}$ is onto. Let $r \in\{0,1, \cdots, n\}$, then $r$ can be written $r=\sum_{i=1}^{k} \alpha_{i} 2^{i-1}$ in the base 2, where $\alpha_{i} \in\{0,1\}$. Set $x=\sum_{i=1}^{k} \alpha_{i} c_{i}$, then $w t(x)=\sum_{i=1}^{k} \alpha_{i} 2^{i-1}=r$

Up an equivalence we have the following result:

Theorem 4 There exists only one distinct weight $\left[2^{k}-1, k\right]$-code, moreover such code is Boolean subring of $\left(\mathbb{F}_{2}^{n},+, \cdot\right)$.

Proof. Let $\mathcal{C}$ be such a code and take code-words $u_{1}, u_{2}, \cdots, u_{k}$, each $u_{i}$ has weight $2^{i-1}$. These are linearly independent and form a basis of $\mathcal{C}$. Next we show that $u_{s} u_{r}=0, \forall s<r$. Otherwise, there exists a least integer $r$ such that $u_{s} u_{r} \neq 0$ for some $s<r$. Since $w t\left(u_{s} u_{r}\right) \leq w t\left(u_{s}\right)$, one have $u_{s} u_{r}=\sum_{i=1}^{r-1} \alpha_{i} u_{i}$. Multiplying by $u_{s}$ yields $u_{s} u_{r}=u_{s}$. Now consider the word $c=u_{r}+u_{s}$,

$w t(c)=w t\left(u_{r}\right)+w t\left(u_{s}\right)-2 w t\left(u_{s} u_{r}\right)=w t\left(u_{r}\right)+w t\left(u_{s}\right)-2 w t\left(u_{s}\right)=2^{r-1}-2^{s-1}$ On the other hand, if we consider $h=u_{s}+u_{s+1}+\cdots+u_{r-1}$, then $w t(h)=\sum_{i=s-1}^{r-2} 2^{i}=2^{r-1}-2^{s-1}$. Thus $u_{s}+u_{s+1}+\cdots+u_{r-1}=u_{r}+u_{s}$ hence $u_{s+1}+\cdots+u_{r-1}+u_{r}=0$ a contradition. This means that $u_{r} u_{s}=0$, if $r \neq s$. Since $u_{r}^{2}=u_{r}$, and $u_{1}, \cdots, u_{k}$ is a basis of $\mathcal{C}$, then $\mathcal{C}$ is a Boolean ring.

Now we define a linear mapping $f: \mathcal{C} \rightarrow \mathcal{D}(k)$ by $f\left(u_{i}\right)=c_{i}$. Then, $f\left(\sum_{i=1}^{k} \alpha_{i} u_{i}\right)=\sum_{i=1}^{k} \alpha_{i} c_{i}$. If $x=\sum_{i=1}^{k} \alpha_{i} u_{i}$, then $w t(f(x))=w t\left(f\left(\sum_{i=1}^{k} \alpha_{i} u_{i}\right)\right)=w t\left(\sum_{i=1}^{k} \alpha_{i} c_{i}\right)=\sum_{i=1}^{k} \alpha_{i} 2^{i-1}=w t(x)$. This implies that $f$ is an isometry between $\mathcal{C}$ and $\mathcal{D}(k)$, and by the extension theorem of MacWilliams, see [3] or [4], there exists a permutation $\sigma \in \mathcal{S}_{n}$, such that $\sigma(\mathcal{C})=\mathcal{D}(k)$.

Example $5 \quad k=3$ and $n=7 \quad\left(2^{3}-1=7\right)$

By using the software Q-extension, see [5] we show, up to equivallence, that among six equivallence classes the unique DW-code $C_{3}$ of parametters $[7,3,1]_{2}$ is the code of generator matrix $G_{3}=\left(\begin{array}{l}0000100 \\ 1110010 \\ 0001001\end{array}\right)$. It is clear that it is equivallent to the code $\mathcal{D}(3)$ of generator matrix $G_{3}^{\prime}=\left(\begin{array}{l}1000000 \\ 0110000 \\ 0001111\end{array}\right)$. Just swap the second and third rows and then apply the permutation $\sigma=(1,5)(2,4)(3,7)$.

Theorem 6 Let $2^{k}=n+1$, Diophantine equations $n=t_{1}+t_{2}+\cdots+t_{k}$ for which

(1) $t_{1}<t_{2}<t_{3}<\cdots<t_{k}$

(2) $t_{i} \neq \sum_{j \in(I\{\{i\})} \varepsilon_{j} t_{j}, \forall i=1,2, \cdots, k, \quad \forall I \subseteq\{1,2, \cdots, k\}, \forall \varepsilon_{j}= \pm 1$, 
have a unique solution which is the $k$-uplet $\left(t_{1}, t_{2}, \cdots, t_{k}\right)=\left(1,2,2^{2}, 2^{3}, \cdots, 2^{k-1}\right)$.

Proof. $\left(1,2,2^{2}, 2^{3}, \cdots, 2^{k-1}\right)$ is clearly a solution of the Diophantine equation which satisfies the conditions (1). Assume that $2^{i}=\sum_{j \in(I \backslash\{i\})} \varepsilon_{j} 2^{j}$ for some $i$ and $I$, then $2^{i}+\sum_{i \in K^{-}} 2^{j}=\sum_{i \in K^{+}} 2^{j}\left(<2^{k}-1\right)$ where $K^{+}=\left\{j \in I \backslash\{i\} / \varepsilon_{j}=1\right\}$ and $K^{-}=\left\{j \in I \backslash\{i\} / \varepsilon_{j}=-1\right\}$. We can assume without loss of generality that $\left\{2^{j} / j \in K^{+}\right\} \cap\left\{2^{j} / j \in K^{-}\right\}=\varnothing$. So by the uniqueness of Development of any integer less than or equal $2^{k}-1$ in binary basis, the equality $2^{i}+\sum_{i \in K^{-}} 2^{j}=\sum_{i \in K^{+}} 2^{j}$ leads to a contradiction. So the solution $\left(1,2,2^{2}, 2^{3}, \cdots, 2^{k-1}\right)$ satisfies the conditions (2).

Conversely, Let $\left(t_{1}, t_{2}, \cdots, t_{k}\right)$ a solution of the equation $n=t_{1}+t_{2}+\cdots+t_{k}$ satisfying (1)-(2). We can take $d_{i}, i=1,2, \cdots, k$ elements of $F_{2}^{n}$ such that $w t\left(d_{i}\right)=t_{i}$ and $d_{1}=(\underbrace{1 \cdots 1}_{t_{1}} \cdots 0)$, $d_{2}=(\underbrace{0 \cdots 0}_{t_{1}} \underbrace{1 \cdots 1}_{t_{2}} \cdots 0), \cdots, d_{k}=(\underbrace{0 \cdots 0}_{t_{1}} \underbrace{0 \cdots 0}_{t_{2}} 0 \cdots 01 \underbrace{1 \cdots 1}_{t_{k}}), d_{i}, i=1,2, \cdots, k$ are linearly independent. The condition (2) means that the code of generator matrix $G=\left(\begin{array}{c}d_{1} \\ \vdots \\ d_{k}\end{array}\right)$ is a dw-code. On after Theorem 1.3, the condition (1) implies that there exists an invertible $k$ by $k$ matrix $A=\left(a_{i, j}\right)_{i, j}$ and a permutation matrix $P_{\sigma}$ such that $A G_{k} P_{\sigma}=G$ where $\sigma \in S_{n}$ and $G_{k}=\left(\begin{array}{ccccccccccccc}1 & 0 & 0 & 0 & 0 & 0 & 0 & 0 & 0 & 0 & \cdots & 0 & 0 \\ 0 & 1 & 1 & 0 & 0 & 0 & 0 & 0 & 0 & 0 & \cdots & 0 & 0 \\ 0 & 0 & 0 & 1 & 1 & 1 & 1 & 0 & 0 & 0 & \cdots & 0 & 0 \\ \vdots & \vdots & \vdots & \vdots & \vdots & \vdots & \vdots & \vdots & \vdots & \vdots & \vdots & \vdots & \vdots \\ 0 & 0 & 0 & 0 & 0 & 0 & 0 & 0 & 0 & 1 & \cdots & 1 & 1\end{array}\right)$ is the generator matrix of the code $\mathcal{D}(k)$. It is clear that $G$ is of the form:

$$
\left(\begin{array}{cccccccc}
a_{1, \sigma(1)} & a_{1, \sigma(2)} & a_{1, \sigma(2)} & \cdots & a_{1, \sigma(k)} & a_{1, \sigma(k)} & \cdots & a_{1, \sigma(k)} \\
a_{2, \sigma(1)} & a_{2, \sigma(2)} & a_{2, \sigma(2)} & \cdots & a_{2, \sigma(k)} & a_{2, \sigma(k)} & \cdots & a_{2, \sigma(k)} \\
\vdots & \vdots & \vdots & \vdots & \vdots & \vdots & \vdots & \vdots \\
a_{k, \sigma(1)} & a_{k, \sigma(2)} & a_{k, \sigma(2)} & \cdots & a_{k, \sigma(k)} & a_{k, \sigma(k)} & \cdots & a_{k, \sigma(k)}
\end{array}\right)
$$

where $a_{i, j}=0$ or $1, \forall i, j$ and $\forall j=1,2,3, \cdots, k$ we have $t_{j}=\sum_{i=1}^{i=k} 2^{i-1} a_{j, \sigma(i)}$. So we have $2^{k}-1=\sum_{j=1}^{j=k} t_{j}=\sum_{j=1}^{j=k}\left(\sum_{i=1}^{i=k} 2^{i-1} a_{j, \sigma(i)}\right)=\sum_{i=1}^{i=k} 2^{i-1}\left(\sum_{j=1}^{j=k} a_{j, \sigma(i)}\right)$, and then we have $\sum_{j=1}^{j=k} a_{j, \sigma(i)}=1, \forall i=1,2, \cdots, k$ by the uniqueness of development of $2^{k}-1$ in binary basis. By (1) we have $\sum_{i=1}^{i=k} 2^{i-1} a_{1, \sigma(i)}<\sum_{i=1}^{i=k} 2^{i-1} a_{2, \sigma(i)}<\cdots<\sum_{i=1}^{i=k} 2^{i-1} a_{k, \sigma(i)}$, then we have: $\forall i, j, a_{i, \sigma(j)}=\delta_{i}^{j} \quad$ (Kronecker symbol).

Since $t_{j}=\sum_{i=1}^{i=k} 2^{i-1} a_{j, \sigma(i)}$, we have $\forall j=1,2, \cdots, k, t_{j}=2^{j-1}$ and finally we have $\left(t_{1}, t_{2}, t_{3}, \cdots, t_{k}\right)=\left(1,2,2^{2}, 2^{3}, \cdots, 2^{k-1}\right)$.

Remark 7 Without the conditions (1) and (2), Diophantine equations have $C_{2^{k}-2}^{k-1}$ different solutions. For all 
$k \geq 3$ note that there is no DW-self-dual code. Indeed, if not, we will have $2^{k}-1 \leq n=2 k$, wich is impossible.

\section{Classification and Automorphism Group of DW-Codes}

\subsection{Automorphism Group: The General Case}

We consider, without loss of generality, that a generator matrix of a DW-code has no zero columns. Indeed, if this is the case, the zero columns are omitted and we consider the obtained DW-code. This assumption is made in the entier paper. We study the automorphism group of DW-codes. We first notice the following:

Proposition 8 Let $\left(u_{1}, u_{2}, \cdots, u_{k}\right)$ any basis of an $[n, k] D W$-code. Then

$$
\operatorname{Aut}(\mathcal{C})=\left\{\sigma \in \mathcal{S}_{n} \mid \sigma\left(u_{i}\right)=u_{i}, \forall i=1, \cdots, k\right\} \text {. }
$$

Moreover, if $G$ any generator matrix of $\mathcal{C}$, then $\sigma$ is an automorphism of $\mathcal{C}$, if and only if, $\sigma$ is an automorphism of the binary matrix $G$.

Proof. Clear.

Proposition 9 The automorphism group of any $D W$-code is nontrivial of even order.

Proof. Let $G$ be a generator matrix of a DW $[n, k]$-code $\mathcal{C}$. We may suppose that all columns of $G$ are nonzero. The $n$ columns of $G$ are taken among a set of $2^{k}-1$ columns. Suppose that all columns of $G$ are distincts, since $n \geq 2^{k}-1$, then the columns of $G$ are the $n=2^{k}-1$ distinct nonzero vectors of $\mathbb{F}_{2}^{k}$ and $\mathcal{C}$ will be the simplex code, which is clearly not DW. This contradiction shows that at least 2 columns of $G$ are identical. Now the transposition of these two columns gives an automorphism of $\mathcal{C}$.

We deduce that the dual code $\mathcal{C}^{\perp}$ of a DW-code has a non-trivial automorphism group and has minimum distance $d^{\perp}=2$.

We consider the general case $2^{k}<n+1$. The action of automorphism group $\operatorname{Aut}(\mathcal{C})$ on the set $\Omega=\left\{c_{1}, c_{2}, \cdots, c_{n}\right\}$ of columns of a generator matrix $G$ defined by: $\sigma\left(c_{i}\right)=c_{\sigma(i)}$ for all $\sigma$ in $\operatorname{Aut}(\mathcal{C})$ and $c_{i}$ in $\Omega$, splits all the columns of $G$ into disjoint orbits. The orbits $O_{1}, O_{2}, \cdots, O_{f}$ each formed of a single column, they are the columns fixed by the group $\operatorname{Aut}(\mathcal{C})$. We set $f=0$ if no orbit is formed of a single column and then it is clear that $0 \leq f<2^{k}-1$ since since $\operatorname{Aut}(\mathcal{C})$ can not be trivial. The $r(\geq 1)$ other orbits are $O_{t_{1}}, O_{t_{2}}, \cdots, O_{t_{r}},\left|O_{t_{i}}\right| \geq 2, \quad i=1,2, \cdots, r$. We set $O_{i}=\left\{c_{i}\right\}, i=1,2, \cdots, f \quad$ if $f \geq 1$ and $O_{t_{i}}=\left\{c_{i}^{(1)}, c_{i}^{(2)}, \cdots, c_{i}^{\left(t_{i}\right)}\right\}, i=1,2, \cdots, r$, therefore, we have precisely $0 \leq f \leq 2^{k}-1-r$.

Up to equivalence, we can consider that the code $\mathcal{C}$ is of generator matrix

$$
G=(\underbrace{c_{1}^{(1)}, c_{1}^{(2)}, \cdots, c_{1}^{\left(t_{1}\right)}}_{O_{t_{1}}}, \cdots, \underbrace{c_{r}^{(1)}, c_{r}^{(2)}, \cdots, c_{r}^{\left(t_{r}\right)}}_{O_{t_{r}}}, \underbrace{c_{1}}_{O_{1}}, \underbrace{c_{2}}_{O_{2}}, \cdots, \underbrace{c_{f}}_{O_{f}})=\left(\begin{array}{c}
d_{1} \\
\vdots \\
d_{k}
\end{array}\right)
$$

$d_{j}=\left(d_{j}^{(i)}\right)_{i}$ rows of $G$, such that $2 \leq t_{1} \leq t_{2} \leq \cdots \leq t_{r}$.

Since $\mathcal{C}$ is a DW-code, then for each $j=1,2, \cdots, k$ for each $\sigma \in \operatorname{Aut}(\mathcal{C})$ we have $\sigma\left(d_{j}\right)=d_{j}$

$\forall j=1,2, \cdots, k$. So $\forall i=1,2, \cdots, n$ we have $d_{j}^{(\sigma(i))}=d_{j}^{(i)}$. We therefore deduce that: $\forall i=1,2, \cdots, r$, $c_{i}^{(1)}=c_{i}^{(2)}=\cdots=c_{i}^{\left(t_{i}\right)}$. So each orbit $O_{t_{i}}$ consists of $t_{i} \geq 2$ equal columns.

The following theorem legitimate the idea of giving a definition to the 3-tuple

$\left(f, k,\left(t_{1}, t_{2}, \cdots, t_{r}\right)\right)$ which we call signature of the DW-code and we denote $\operatorname{sign}(\mathcal{C})=\left(f, k,\left(t_{1}, t_{2}, \cdots, t_{r}\right)\right)$. We give here the full classification of such a code in several cases.

Theorem 10 If two $D W$-codes $\mathcal{C}$ and $\mathcal{C}^{\prime}$ are equivalent then they have the same signatures: $\operatorname{sign}(\mathcal{C})=\operatorname{sign}\left(\mathcal{C}^{\prime}\right)$.

Proof. Let $\mathcal{C}$ and $\mathcal{C}^{\prime}$ two equivalent DW-codes of parameters $[n, k]$. So $\exists \sigma \in S_{n}$ such as $\sigma(\mathcal{C})=\mathcal{C}^{\prime}$. 
We have $\operatorname{Aut}\left(\mathcal{C}^{\prime}\right)=\sigma \operatorname{Aut}(\mathcal{C}) \sigma^{-1}$. Let $G$ be a generator matrix of the code $\mathcal{C}$. Under the action of the automorphism group $\operatorname{Aut}(\mathcal{C})$ we can assume that $G$ is of the form $G=\left(O_{t_{1}}, \cdots, O_{t_{r}}, O_{1}, O_{2}, \cdots, O_{f}\right)$ where $O_{t_{i}}=\left\{c_{s_{i-1}+1}, \cdots, c_{s_{i}}\right\}, \quad s_{i}=\sum_{j=1}^{j=i} t_{j} \quad \forall i=1,2, \cdots, t_{r}$ and $O_{i}=\left\{c_{s_{r}+i}\right\} \quad \forall i=1,2, \cdots, f$.

So we have $O_{t_{i}}=\left\{c_{s_{i-1}+1}, \cdots, c_{s_{i}}\right\}=\left\{c_{\sigma\left(s_{i}\right)} / \sigma \in \operatorname{Aut}(\mathcal{C})\right\}=\left\{c_{\sigma^{-1} \rho \sigma\left(s_{i}\right)} / \rho \in \operatorname{Aut}\left(\mathcal{C}^{\prime}\right)\right\}$ and then $\sigma\left(O_{t_{i}}\right)=\left\{c_{\rho \sigma\left(s_{i}\right)} / \rho \in \operatorname{Aut}\left(\mathcal{C}^{\prime}\right)\right\}=O_{t_{i}}^{\prime}$ which is an orbit of the column $c_{\sigma\left(s_{i}\right)}$ under the action of $\operatorname{Aut}\left(\mathcal{C}^{\prime}\right)$ on the generator matrix $G^{\prime}=\sigma(G)$ of the code $\mathcal{C}^{\prime}$. similarly we have $\sigma\left(O_{i}\right)=\left\{c_{\rho \sigma\left(s_{r}+i\right)} / \rho \in \operatorname{Aut}\left(\mathcal{C}^{\prime}\right)\right\}=O_{i}^{\prime}$ which is an orbit of the column $C_{\sigma\left(s_{r}+i\right)}$ under the action of $\operatorname{Aut}\left(\mathcal{C}^{\prime}\right)$ on the generator matrix $G^{\prime}$. Thus $G$ and $G^{\prime}$ have the same number of ponctual orbits, the same number of non-ponctual orbits and the two orbits $O_{t_{i}}$ and $O_{t_{i}}^{\prime}$ on the one hand and $O_{i}$ and $O_{i}^{\prime}$ on the other hand have the same number of columns. we conclude that the two codes $C$ and $C^{\prime}$ have the same Signature.

\subsection{Classification}

3.2.1. Case 1: $f=0$ and $k=r$

We have

$$
G=(\underbrace{c_{1}^{(1)}, c_{1}^{(2)}, \cdots, c_{1}^{\left(t_{1}\right)}}_{o_{t_{1}}}, \cdots, \underbrace{c_{k}^{(1)}, c_{k}^{(2)}, \cdots, c_{k}^{\left(t_{k}\right)}}_{o_{t_{k}}})=\left(\begin{array}{c}
d_{1} \\
\vdots \\
d_{k}
\end{array}\right)
$$

Theorem 11 If $\mathcal{C}$ is an $[n, k]_{2} \quad D W$-code without punctual orbits $(f=0)$ and if the number of non punctual orbits is equal to the dimension of the DW-code $(r=k)$ then the code $\mathcal{C}$ is equivallent to a DWcode of generator matrix

$$
G=G_{k}\left(t_{1}, t_{2}, \cdots, t_{k}\right)=\left(\begin{array}{c}
\underbrace{1 \cdots 1000 \cdots 0}_{t_{1}} \cdots 0 \\
0 \cdots 0 \underbrace{1 \cdots 1}_{t_{2}} \cdots 0 \cdots 0 \\
\vdots \\
000000 \cdots 00 \underbrace{1 \cdots 1}_{t_{k}}
\end{array}\right) \text { whith } 2 \leq t_{1}<t_{2}<\cdots<t_{k} \text { and } t_{1}=d \text { is the minimal distance of }
$$

$C_{k}$.

Proof. After a series of permutations and elementary operations on rows of $G$ we can make the first line of

$$
\overbrace{11 \cdots 1}^{O_{1} \cdots 1}
$$

the first orbit formed only by ones and all other rows are null $\begin{gathered}00 \cdots 0 \\ \vdots\end{gathered}$ all other bits of the first row of the

$$
00 \cdots 0
$$

generator matrix are zero. Otherwise the first line of another orbit $O_{t_{s}}$ will be formed only by $1 \mathrm{~s}$. And a series of permutations and elementary row operations can make null all the other rows of this orbit so $O_{t_{1}} \cap O_{t_{s}} \neq \varnothing$. This is a contradiction since two orbits are disjoint. We obtain a generator matrix of an equivalent code denoted by the same sign $G_{k}=\left(\begin{array}{c|c}11 \cdots 1 & 00 \cdots 0 \\ 0 & \\ \vdots & G_{k}^{0} \\ 0 & \end{array}\right)$. It is easy to see that $G_{k}^{0}$ is a generator matrix of a DW-code 
without punctual orbits $\left(f_{G_{k}^{0}}=0\right)$ and the number of orbits is equal to the dimension of this DW-code $\left(r_{G_{k}^{0}}=k-1\right)$ and This allows for reasoning by induction. We obtain a generator matrix of an equivalent code

$$
G_{k}\left(t_{1}, t_{2}, \cdots, t_{k}\right)=\left(\begin{array}{c}
1 \cdots 1000 \cdots 0 \cdots 0 \\
\underbrace{1}_{t_{1}} \ldots 0000 \\
0 \underbrace{1 \cdots 1}_{t_{2}} \cdots 0 \cdots 0 \\
\vdots \\
000000 \cdots 00 \underbrace{1 \cdots 1}_{t_{k}}
\end{array}\right) .
$$

It is clear that we have $w t\left(d_{1}\right)=t_{1}=d, w t\left(d_{2}\right)=t_{2}, \cdots, w t\left(d_{k}\right)=t_{k}$ and $2 \leq t_{1}<t_{2}<\cdots<t_{k}, \quad\left(t_{1} \geq 2\right.$ since $t_{1}=1$ implies the existence of a punctual orbit $)$.

Remark 12 In this case, up to equivallence, each $[n, k]_{2}$ DW-code admits the system $\left\{d_{1}, d_{2}, \cdots, d_{k}\right\}$ as orthogonal basis: $d_{1}=(\underbrace{1 \cdots 10 \cdots 0}_{t_{1}}), d_{2}=(\underbrace{0 \cdots 0}_{t_{1}} \underbrace{1 \cdots 10 \cdots 0}_{t_{2}}), \cdots, d_{2}=(\underbrace{0 \cdots 0}_{t_{1}} \underbrace{0 \cdots 0}_{t_{2}} 0 \cdots 0 \underbrace{1 \cdots 1}_{t_{k}})$ such as $w t\left(d_{1}\right)=t_{1}=d, w t\left(d_{2}\right)=t_{2}, \cdots, w t\left(d_{k}\right)=t_{k}$ and $2 \leq t_{1}<t_{2}<\cdots<t_{k}$.

Example 13 Consider the $[15,3,2]_{2}$ DW-code of generator matrix

$\left(\begin{array}{l}100000000000100 \\ 011111111000010 \\ 000000000111001\end{array}\right)$. It is equivallente to the code of generator matrix $\left(\begin{array}{l}110000000000000 \\ 001111000000000 \\ 0000001111111111\end{array}\right)$

$t_{1}=2, t_{2}=4, t_{3}=9, f=0, r=k=3$

Corollary 14 Let two $[n, k]_{2} \quad D W$-codes $\mathcal{C}$ and $\mathcal{C}^{\prime}$ without punctual orbits and the number of their orbits is equal to their dimension. Then the codes $\mathcal{C}$ and $\mathcal{C}^{\prime}$ are equivallent if and only if $\operatorname{sign}(\mathcal{C})=\operatorname{sign}\left(\mathcal{C}^{\prime}\right)$.

The converse of Theorem 11 is true under an additional condition.

Theorem 15 Let $\mathcal{C}$ an $[n, k]_{2}$ code of generator matrix $G_{k}\left(t_{1}, t_{2}, \cdots, t_{k}\right)$ (as in the last remark). If:

(1) $2 \leq t_{1}<t_{2}<\cdots<t_{k}$

(2) $\forall I \subseteq\{1,2, \cdots, k\}, \forall i=1,2, \cdots, k, \forall \varepsilon_{j}= \pm 1$ we have $t_{i} \neq \sum_{j \in(I \backslash\{i\})} \varepsilon_{j} t_{j}$

then $\mathcal{C}$ is a DW-code of minimum distance $d=t_{1}$ for which $f=0$ and $r=k$.

Proof. Clear.

Corollary 16 The number of equivalence classes of $[n, k, d]_{2}$ DW-codes such as $f=0, k=r$ and $2^{k}<n+1$ equals the number of solutions $\left(t_{1}, t_{2}, t_{3}, \cdots, t_{k}\right)$ of the Diophantine equations $n=t_{1}+t_{2}+t_{3}+\cdots+t_{k}$ satisfying the following conditions

(1) $d=t_{1}<t_{2}<t_{3}<\cdots<t_{k}$

(2) $t_{i} \neq \sum_{j \in(I \backslash\{i\})} \varepsilon_{j} t_{j}, \forall i=1,2, \cdots, k, \forall \varepsilon_{j}= \pm 1, \forall I \subseteq\{1,2, \cdots, k\}$.

Proof. Let the application that maps each equivalence class represented by the matrix $G_{k}\left(t_{1}, t_{2}, \cdots, t_{k}\right)$ to the $t$-tuple $\left(t_{1}, t_{2}, \cdots, t_{k}\right)$ solution of the Diophantine equation as described in Theorem 11. This application is clearly a bijection between the set of equivalence classes and the set of solutions of the Diophantine equation satisfying conditions (1) and (2).

3.2.2. Case 2: $f \neq 0$ and $k=r$

Theorem 17 If $\mathcal{C}$ is an $[n, k]_{2}$ DW-code with $f$ punctual orbits $(f \neq 0)$ and if the number of non punctual orbits is equal to the dimension of the DW-code $(r=k)$ then the code $C$ is equivallent to the 
$D W$-code of generator matrix $G=\left(\begin{array}{cccc|c|c}\underset{1 \cdots 1}{t_{1}} & 0 \cdots 0 & \cdots & 0 \cdots 0 & \mid & c_{1} c_{2} \cdots c_{f} \\ 0 \cdots 0 & \underbrace{1 \cdots 1}_{t_{2}} & \cdots & 0 \cdots 0 & \vdots \\ 0 \cdots 0 & 0 \cdots 0 & \cdots & 0 \cdots 0 & \vdots \\ \vdots & \vdots & \vdots & \vdots & \vdots \\ 0 \cdots 0 & 0 \cdots 0 & \cdots & 0 \cdots 0 & \vdots \\ 0 \cdots 0 & 0 \cdots 0 & \cdots & \underbrace{1 \cdots 1}_{t_{k}} & \vdots\end{array}\right)$ with $1 \leq f \leq 2^{k}-1-k$ and $2 \leq t_{1} \leq t_{2} \leq \cdots \leq t_{k}$

Example 18 Consider the $[15,3,4]_{2} \quad D W$-code $\mathcal{C}_{1}$ of generator matrix

$G_{1}=\left(\begin{array}{llll}111 & 0000000 & 000 & 10 \\ 111 & 1111111 & 000 & 01 \\ 111 & 0000000 & 111 & 01\end{array}\right)$. It is equivallente to the code $\mathcal{C}_{2}$ of generator matrix

$G_{2}=\left(\begin{array}{llll}111 & 000 & 0000000 & \stackrel{F}{10} \\ 000 & 111 & 0000000 & 11 \\ 000 & 000 & 1111111 & 11\end{array}\right) t_{1}=3, t_{2}=3, t_{3}=7, f=2, \quad r=k=3$

We have $\operatorname{sign}\left(\mathcal{C}_{1}\right)=\operatorname{sign}\left(\mathcal{C}_{2}\right)$ since $\mathcal{C}_{1}$ and $\mathcal{C}_{2}$ are equivallente.

The converse of this theorem is true under an additional condition. Let $\mathcal{C}$ an $[n, k]_{2}$ of generator matrix $\left(\begin{array}{ccc|c}\underbrace{1 \cdots 1}_{t_{1}} & & & c_{1} c_{2} \cdots c_{f} \\ & \ddots & & \vdots \\ & & \underbrace{1 \cdots 1}_{t_{k}} & \vdots\end{array}\right)$

with: $1 \leq f \leq 2^{k}-1-k$ and $2 \leq t_{1} \leq t_{2} \leq \cdots \leq t_{k} . c_{1}, c_{2}, \cdots, c_{f}$ are $\mathrm{f}$ different columns which are also different from all unitary columns $(1,0, \cdots, 0)^{\mathrm{T}},(0,1,0, \cdots, 0)^{\mathrm{T}}, \cdots,(0, \cdots, 0,1)^{\mathrm{T}}$.

For each $I \subseteq\{1,2, \cdots, k\}$, denote by $\omega_{I, \epsilon}$ the weight of the sum of all the jth rows where $j \in I \backslash\{i\}$ of the $k \times f$ matrix

$$
A=\left(\begin{array}{cccccc}
\varepsilon_{1} & 0 & 0 & 0 & \cdots & 0 \\
0 & \varepsilon_{2} & 0 & 0 & \cdots & 0 \\
0 & 0 & \varepsilon_{3} & 0 & \cdots & 0 \\
\vdots & \vdots & \vdots & \vdots & \vdots & \vdots \\
0 & 0 & 0 & 0 & \cdots & \varepsilon_{k}
\end{array}\right)\left(c_{1}, c_{2}, \cdots, c_{f}\right) \quad \varepsilon_{j}= \pm 1, \quad \varepsilon=\left(\varepsilon_{j}\right)_{j}
$$

For all $i=1,2, \cdots, k$ denote by $\alpha_{i}$ the weight of the ith row of the $k \times f$ matrix $\left(c_{1}, c_{2}, \cdots, c_{f}\right)$. So by setting the numbers $\rho_{I, i, \epsilon}=\omega_{I, \epsilon}-\alpha_{i}$ we have the following result, let $\mathcal{C}$ an $[n, k]$ code of generator matrix $G$ as described in theorem 17 we have:

Theorem 19 If for all $I \subseteq\{1,2, \cdots, k\}$, for all $i \in I$ and for all $\epsilon=\left(\varepsilon_{j}\right)_{j}, \varepsilon_{j}= \pm 1$ we have $t_{i} \neq\left(\sum_{j \in(I \backslash\{i\})} \varepsilon_{j} t_{j}\right)+\rho_{I, i, \epsilon}$ then the code $\mathcal{C}_{k}$ is a DW-code.

Let $C_{k}$ an $[n, k]_{2}$ DW-code such as $2^{k}<n+1, r=k$ and $1 \leq f \leq 2^{k}-1-k$. We have $n(f)=C_{2^{k}-1-k}^{f}$ different way to the choice of $f$ fixed columns. For each value of $f$ and for the $s_{f}$-th choice of $f$ fixed 
columns we denote by $N\left(f, s_{f}\right), 1 \leq s_{f} \leq C_{2^{k}-1-k}^{f}$ the number of solutions of the Diophantine equations $n-f=t_{1}+t_{2}+t_{3}+\cdots+t_{k}$ which satisfy the following conditions

(1) $2 \leq t_{1} \leq t_{2} \leq t_{3} \leq \cdots \leq t_{k}$

(2) $t_{i} \neq\left(\sum_{j \in(I \backslash\{i\})} \varepsilon_{j} t_{j}\right)+\rho_{I, i, \epsilon} \quad \forall i=1,2, \cdots, k, \forall I \subseteq\{1,2, \cdots, k\}, \forall \epsilon=\left(\varepsilon_{j}\right)_{j}, \quad \varepsilon_{j}= \pm 1$

So we have the following result.

\section{Theorem 20}

1) The number of equivalence classes of $[n, k]$ DW-codes with $2^{k}<n+1$ and a given $f$ such that $1 \leq f \leq 2^{k}-1-k$ and $k=r$ equals the number $\sum_{s_{f}=1}^{s_{f}=n(f)} N\left(f, s_{f}\right)$.

2) The number of equivalence classes of $[n, k]_{2}$ DW-codes with $2^{k}<n+1, f \neq 0$ and $k=r$ equals the number $\sum_{f=1}^{f=2^{k}-1-k}\left(\sum_{s_{f}=1}^{s_{f}=n(f)} N\left(f, s_{f}\right)\right)$.

Example 21 By using the result of the last theorem and the Q-extension software, We show that there exist Only $4[11,3]_{2}$ DW-code up to equvallence verifying $r=k=3$. Indeed $1 \leq f \leq 2^{3}-1-3=4$ and we have:

- For $f=1$ the set of possible columns taken in the following order are:

$$
\begin{array}{rllll}
c_{1} & c_{2} & c_{3} & c_{4} \\
1 & 1 & 0 & 1 \\
1 & 0 & 1 & 1 \\
0 & 1 & 1 & 1 \\
N(1,1)=N(1,3)= & N(1,4)=1, \quad N(1,2)=0
\end{array}
$$

So the number of DW-codes with $2^{k}<n+1, f=1$ and $k=r$ is $\sum_{i=1}^{i=4} N(1, i)=3$.

- For $f=2$ the set of possible columns taken in the following order are:

$$
\begin{aligned}
& \overbrace{\left[c_{1}, c_{2}\right]}^{1 \text { th }} \frac{\overbrace{\left[c_{1}, c_{3}\right]}^{2 \text { th }}}{\overbrace{\left[c_{1}, c_{4}\right]}^{3 \text { th }}} \overbrace{\left[c_{2}, c_{3}\right]}^{\text {4th }} \overbrace{\left[c_{2}, c_{4}\right]}^{\text {5th }} \overbrace{\left[c_{3}, c_{4}\right]}^{6 \text { th }} \\
& \begin{array}{llllll}
11 & 10 & 11 & 10 & 11 & 01
\end{array} \\
& \begin{array}{lllllll}
10 & 11 & 11 & 01 & 01 & 11
\end{array} \\
& \begin{array}{llllll}
01 & 01 & 01 & 11 & 11 & 11
\end{array} \\
& N(2,1)=N(2,2)=N(2,3)=N(2,4)=N(2,5)=N(2,6)=0
\end{aligned}
$$

So there is no DW-codes with $2^{k}<n+1, f=2$ and $k=r$ since $\sum_{i=1}^{i=6} N(2, i)=0$.

- For $f=3$ the set of possible columns taken in the following order are:

$$
\begin{array}{ccccc}
\frac{1 \text { th }}{\left[c_{1} c_{2} c_{3}\right]} & \frac{2 \text { th }}{\left[c_{1} c_{2} c_{4}\right]} & \frac{3 \text { th }}{\left[C_{1} c_{3} c_{4}\right]} & \frac{4 \text { th }}{\left[C_{2} c_{3} c_{4}\right]} \\
110 & 111 & 101 & 101 \\
101 & 101 & 111 & 011 \\
011 & 011 & 011 & 111 \\
N(3,1)=N(3,2)=N(3,3)=N(3,4)=0
\end{array}
$$

So there is no DW-codes avec $2^{k}<n+1, f=3$ and $k=r$ since $\sum_{i=1}^{i=4} N(3, i)=0$. 
- For $f=4$ the set of possible columns taken in the following order are:

$$
\begin{gathered}
\underset{\left[c_{1} c_{2} c_{3} c_{4}\right]}{1 \text { th }} \\
1101 \\
1011 \\
0111 \\
N(4,1)=0, N(4,2)=0, N(4,3)=1, N(4,4)=0 .
\end{gathered}
$$

So there is one DW-codes such as $2^{k}<n+1, f=3$ and $k=r$ since $\sum_{i=1}^{i=1} N(4, i)=1$.

We deduce that there is only four $[11,3]_{2}$ DW-codes, among 98 equivalence classes, satisfying $r=k=3$ since

$$
\sum_{f=1}^{f=4}\left(\sum_{i=1}^{i=n(f)} N(f, i)\right)=\sum_{i=1}^{i=4} N(1, i)+\sum_{i=1}^{i=6} N(2, i)+\sum_{i=1}^{i=4} N(3, i)+\sum_{i=1}^{i=1} N(4, i)=4
$$

3.2.3. Case 3: $f=0$ and $k \neq r$

We have necessarily $k<r$.

Theorem 22 If $\mathcal{C}$ is an $[n, k]_{2}$ DW-code without punctual orbits $(f=0)$ and if the number of non punctual orbits is different from the dimension of the DW-code $(r \neq k)$ then

$k<r$ and the code $\mathcal{C}$ is equivallent to the DW-code of generator matrix

$$
\left(\begin{array}{ccccccc}
\underbrace{1 \cdots 1}_{t_{1}} & & & *_{\ldots \ldots *}^{t_{k+1}} & \ldots & *_{t_{r}}^{t_{r}} \\
& \ddots & & \vdots & \ddots & \vdots \\
& & \underbrace{1 \cdots 1}_{t_{k}} & & & &
\end{array}\right)
$$

with $2 \leq t_{1} \leq t_{2} \leq \cdots \leq t_{k}$.

Example 23 The $[15,3,4]_{2}$ DW-code of generator matrix $\left(\begin{array}{l}111000000000100 \\ 000111111110010 \\ 000111100001001\end{array}\right)$ is equivallent to the code of generator matrix $\left(\begin{array}{ll}11000000000 & 1111 \\ 00111100000 & 0000 \\ 00000011111 & 1111\end{array}\right) f=0, k=3, r=4, t_{1}=2, t_{2}=4, t_{3}=5, t_{4}=4$.

In this case two DW-codes with the same signature are not necessarily equivalent as shown in the following example:

Example 24 Let $\mathcal{C}_{1}$ the DW-code of generator matrix $G_{1}$ and $\mathcal{C}_{2}$ the DW-code of generator matrix $G_{2}$ such as $\mathcal{C}_{1}$ and $\mathcal{C}_{2}$ are not equivalent and

$$
\begin{aligned}
G_{1} & =\left(\begin{array}{l}
110000000000001 \\
111111111100000 \\
000000000011110
\end{array}\right), \\
G_{2} & =\left(\begin{array}{l}
110000000000001 \\
111111111100000 \\
110000000011110
\end{array}\right) .
\end{aligned}
$$


We have $\operatorname{sign}\left(\mathcal{C}_{1}\right)=\operatorname{sign}\left(\mathcal{C}_{2}\right)=(1,3,(2,4,8))$.

3.2.4. Case 4: $f \neq 0$ and $k \neq r$

We can have two cases $k<r$ or $r<k$

Theorem 25 If $\mathcal{C}$ is an $[n, k]_{2}$ DW-code with $f$ punctual orbits $(f \neq 0)$ and if the number of non punctual orbits is greater than the dimension of the DW-code $(r>k)$ then the code $\mathcal{C}$ is equivallent to the

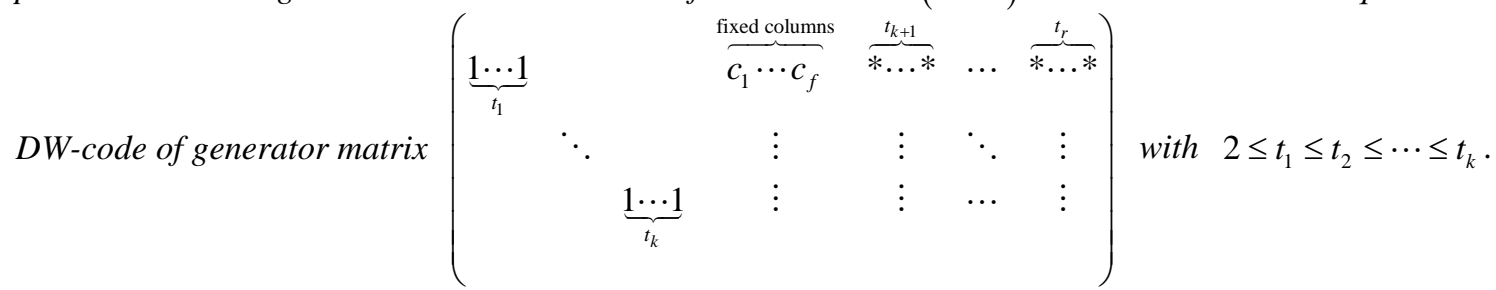

Example 26 The $[15,3,4]_{2}$ DW-code of generator matrix $\left(\begin{array}{l}111000000000100 \\ 111111111110010 \\ 11111000001001\end{array}\right)$ is equivallent to the code of generator matrix $\left(\begin{array}{lll}1100000 & 1111111 & 0 \\ 0011000 & 1111111 & 1 \\ 0000111 & 0000000 & 0\end{array}\right) t_{1}=2, t_{2}=2, t_{3}=3, t_{4}=7, f=1, k=3, \quad r=4$

Theorem 27 If $C$ is an $[n, k]_{2} \quad D W$-code with $f$ punctual orbits $(f \neq 0)$ and if the number of non punctual orbits is lower than the dimension of the DW-code $(r<k)$ then the code $C$ is equivallent to the $D W$-code of generator matrix

$$
\left(\begin{array}{cccc}
\underbrace{1 \cdots 1}_{t_{1}} & & & \overbrace{c_{1} c_{2} \cdots c_{f}}^{\text {fixed columns }} \\
& \ddots & & \vdots \\
& & \underbrace{1 \cdots 1}_{t_{r}} & \vdots \\
000 & \cdots & 000 & \vdots \\
\vdots & \vdots & \vdots & \vdots \\
000 & \cdots & 000 & \vdots
\end{array}\right)
$$

with $2 \leq t_{1} \leq t_{2} \leq \cdots \leq t_{r}$.

Example 28 The $[15,3,4]_{2} \quad D W$-code of generator matrix $\left(\begin{array}{l}110000000000100 \\ 111111110000010 \\ 100000001111001\end{array}\right)$ is equivallent to the code of generator matrix $\left(\begin{array}{ll}111110000000 & 100 \\ 000001111111 & 110 \\ 000000000000 & 111\end{array}\right) t_{1}=5, t_{2}=7, f=3, k=3, \quad r=2$.

Remark 29 Self-orthogonality.

A code which is equivalent to a self-orthogonal code is also self-orthogonal. The property of self- orthogonality is then an invariant of the equivalence of codes. We then have the following points:

- If $f \neq 0$ or $(f=0$ and $r \neq k$ )then, up to equivalence, a generator matrix of the code is of the form $G=[T D]$ where $D$ is not an empty submatrix. If the code is self-othogonal then $G G^{\mathrm{T}}=0$. So

$[T D][T D]^{\mathrm{T}}=0$ and then we have:

- $f \neq 0$ and $r>k$ and then $D D^{\mathrm{T}}=\operatorname{diag}\left(t_{1}[2], t_{2}[2], \cdots, t_{k}[2]\right)$ 
- $f \neq 0$ and $r<k$ then $D D^{\mathrm{T}}=\left(\begin{array}{ll}B & 0 \\ 0 & 0\end{array}\right)$ where $B=\operatorname{diag}\left(t_{1}[2], t_{2}[2], \cdots, t_{r}[2]\right)$.

- $f \neq 0$ and $r=k$ then $D D^{\mathrm{T}}=\operatorname{diag}\left(t_{1}[2], t_{2}[2], \cdots, t_{k}[2]\right)$

- finally $f=0$ and $r \neq k$ and then $D D^{\mathrm{T}}=\operatorname{diag}\left(t_{1}[2], t_{2}[2], \cdots, t_{k}[2]\right)$

- If $f=0$ and $r=k$ the code $C$ is self-orthogonal if and only if $t_{i} \equiv 0[\bmod 2]$ for all $i=1,2, \cdots, k$.

\subsection{Determination of the Automorphism Group}

Theorem 30 The automorphism group of a $[n, k]$ DW-code of signature $\left(f, k,\left(t_{1}, t_{2}, \cdots, t_{r}\right)\right)$ is isomorphic to the group direct product $\prod_{i=1}^{r} \mathcal{S}_{t_{i}}$.

Proof. Let $G$ be a generator matrix of the code $\mathcal{C}$. We can assume that $G$ is of the form $G=\left(O_{t_{1}}, \cdots, O_{t_{r}}, O_{1}, O_{2}, \cdots, O_{f}\right)$ where $O_{t_{i}}=\left\{c_{s_{i-1}+1}, \cdots, c_{s_{i}}\right\}, \quad s_{i}=\sum_{j=1}^{j=i} t_{j} \quad \forall i=1,2, \cdots, t_{r}$ and $O_{i}=\left\{c_{s_{r}+i}\right\}$ $\forall i=1,2, \cdots, f$.

For $i=1, \cdots, r$, let $E_{i}=\left\{s_{i-1}+1, \cdots, s_{i}\right\}$, let $E_{s_{r}+i}=\left\{s_{r}+i\right\}$ for all $i=1,2, \cdots, f$. Clearly, the subsets $E_{1}, \cdots, E_{n}$ form a partition of $\{1,2, \cdots, n\}$. Now let $G_{i}=\left\{\sigma \in S_{n} / \sigma\left(E_{i}\right) \subset E_{i}\right.$ and $\left.\forall j \neq i, \sigma(x)=x, \forall x \in E_{j}\right\}$.

Clearly the $G_{i}$ are subgroups of $\mathcal{S}_{n}$ and each is isomorphic to $\mathcal{S}_{t_{i}}$ and $G_{i}=\{$ id $\}$ for all $i=s_{r}+1, \cdots, s_{r}+f=n$. Since forall $\sigma \in G_{i}, \sigma\left(c_{j}\right)=c_{j}$, it follows that the $G_{i}$ are subgroups of Aut $(\mathcal{C})$. Now we are going to show that $\operatorname{Aut}(\mathcal{C})$ is the inner direct product $G_{1} G_{2} \cdots G_{k}$

If $i \neq j$, and $\sigma \in G_{i}, \tau \in G_{j}$, then $\sigma \tau=\tau \sigma$.

Let $\sigma_{1} \sigma_{1} \cdots \sigma_{k}=I$, then applying this equality to each $E_{i}$ yields $\sigma_{i}=I, \forall i$.

Now let $\sigma \in \operatorname{Aut}(\mathcal{C})$. Since $\sigma\left(c_{i}\right)=c_{i}$, the $E_{i}$ are globally invariant under $\sigma$. Let $\sigma_{i}$ the permutation defined by $\sigma_{i}(x)=\sigma(x)$, if $x \in E_{i}$, and $\sigma_{i}(x)=x$ elswhere. Then it is clear that $\sigma=\sigma_{1} \sigma_{2} \cdots \sigma_{r}$, and this finishes the proof.

\section{Example 31}

- Consider the $[15,3,2]_{2}$ DW-code of generator matrix $\left(\begin{array}{l}100000000000100 \\ 011111111000010 \\ 000000000111001\end{array}\right)$. It is equivallente to the code of generator matrix $\left(\begin{array}{l}110000000000000 \\ 001111000000000 \\ 000000111111111\end{array}\right) t_{1}=2, t_{2}=4, t_{3}=9, f=0, r=k=3$,

$\operatorname{Aut}(\mathcal{C})=S_{2} \cdot S_{4} \cdot S_{9} \quad$ and $\quad|\operatorname{Aut}(C)|=(2 !) \times(4 !) \times(9 !)$

- Consider the $[15,3,5]_{2}$ DW-code of generator matrix $\left(\begin{array}{l}111100000000100 \\ 111111111111010 \\ 111011100000001\end{array}\right)$. It is equivallente to the code of generator matrix $\left(\begin{array}{ll}111000000000 & 110 \\ 000111000000 & 111 \\ 000000111111 & \frac{101}{\text { fixed }}\end{array}\right) t_{1}=3, t_{2}=3, t_{3}=6, f=3, r=k=3$

$\operatorname{Aut}(\mathcal{C})=S_{2} \cdot S_{4} \cdot S_{9} \quad$ and $\quad|\operatorname{Aut}(C)|=(2 !) \times(4 !) \times(9 !)$ 


\section{Acknowledgements}

The authors would like to thank the refrees for their helpful suggestions and remarks.

\section{References}

[1] Bouyukliev, I. (2007) About the Code Equivalence, Advances in Coding Theory and Cryptology. In: Shaska, T., Huffman, W.C., Joyner, D. and Ustimenko, V., Eds., Series in Coding Theory and Cryptology, World Scientific Publishing, Vol. 3, 126-151.

[2] MacWilliams, F.J. and Sloane, N.J.A. (1977) The Theory of Error-Correcting Codes. Elsevier-North-Holland, Amsterdam.

[3] MacWilliams, F.J. (1962) Combinatorial Problems of Elementary Abellan Groups. Ph.D. Dissertation, Harvard University, Cambridge.

[4] Bogart, K., Goldberg, D. and Gordon, J. (1978) An Elementary Proof of the MacWilliams Theorem on Equivalence of Codes. Information and Control, 37, 19-22. http://dx.doi.org/10.1016/S0019-9958(78)90389-3

[5] Bouyukliev, I.G. (2007) What Is Q-Extension? Serdica Journal of Computing, 1, 115-130. 